\title{
Escherichia coli-Derived Outer Membrane Vesicles Deliver Galactose- 1-Phosphate Uridyltransferase and Yield Partial Protection against Actinobacillus pleuropneumoniae in Mice
}

\author{
Keji Quan ${ }^{1 \dagger}$, Zhuang Zhu ${ }^{1 \dagger}$, Sanjie Cao ${ }^{1,2,3 * \dagger}$, Fei Zhang ${ }^{1}$, Chang Miao ${ }^{1}$, Xintian Wen ${ }^{1,2}$, Xiaobo Huang ${ }^{1,2}$, \\ Yiping Wen ${ }^{1,2}$, Rui $\mathrm{Wu}^{1,2}$, Qigui Yan ${ }^{1,2}$, Yong Huang ${ }^{1,3}$, Xiaoping $\mathrm{Ma}^{1,3}$, Xinfeng Han $^{1,3}$, and Qin Zhao ${ }^{1,2 *}$ \\ ${ }^{1}$ Research Center of Swine Disease, College of Veterinary Medicine, Sichuan Agricultural University, Chengdu 611130, P.R. China \\ ${ }^{2}$ Sichuan Science-observation Experimental Station of Veterinary Drugs and Veterinary Diagnostic Technology, Ministry of Agriculture, \\ Chengdu, P.R. China \\ ${ }^{3}$ National Teaching and Experimental Center of Animal, Sichuan Agricultural University, Chengdu 611130, P.R. China
}

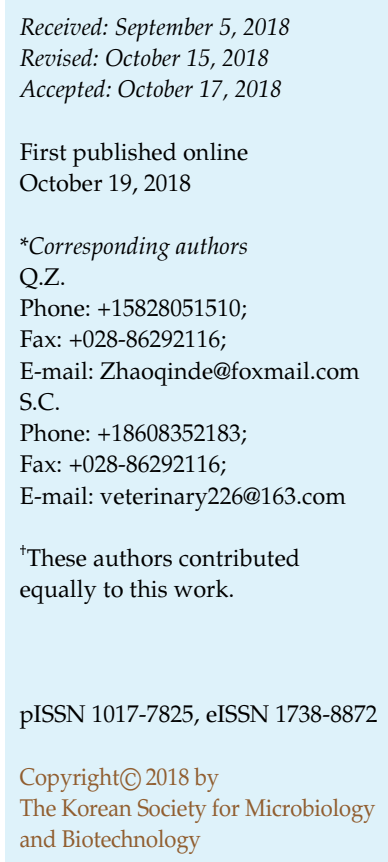

In our previous studies, we have identified several in vivo-induced antigens and evaluated their potential as subunit vaccine candidates in a murine model, in which the recombinant protein GalT showed the most potent immunogenicity and immunoprotective efficacy against Actinobacillus pleuropneumoniae. To exploit a more efficient way of delivering GalT proteins, in this study, we employed the widely studied E. coli outer membrane vesicles (OMVs) as a platform to deliver GalT protein and performed the vaccine trial using the recombinant GalTOMVs in the murine model. Results revealed that GalT-OMVs could elicit a highly-specific, IgG antibody titer that was comparable with the adjuvant GalT group. Significantly higher lymphocyte proliferation and cytokines secretion levels were observed in the GalT-OMVs group. $87.5 \%$ and $50 \%$ of mice were protected from a lethal dose challenge using A. pleuropneumoniae in active or passive immunization, respectively. Histopathologic and immunohistochemical analyses showed remarkably reduced pathological changes and infiltration of neutrophils in the lungs of mice immunized with GalT-OMVs after the challenge. Taken together, these findings confirm that OMVs can be used as a platform to deliver GalT protein and enhance its immunogenicity to induce both humoral and cellular immune responses in mice.

Keywords: Actinobacillus pleuropneumoniae, GalT protein, OMVs, immunoprotective efficacy

\section{Introduction}

Actinobacillus pleuropneumoniae (APP) is the causative agent of porcine contagious pleuropneumonia (PCP), a severe respiratory disease that has caused serious economic losses in the swine industry worldwide [1-4]. To date, 18 serotypes have been identified depending on the antigenic properties of capsular polysaccharides and lipopolysaccharides $[5,6]$. Although vaccines and antibiotics are two major methods of controlling APP infection, increasing antibiotic resistance and growing pressure induced by antibiotics have restricted their clinical application [2]. Therefore, the development of effective vaccines is necessary to prevent this important disease.

Although various vaccines such as whole-cell bacterins, recombinant subunit vaccines, and live attenuated vaccines had been studied [7-10], complete protection could not be achieved yet to control the infection. Among traditional vaccine designs, protein subunit vaccines have been considered to be the most attractive vaccine candidate due to the minimal side effects in comparison with the live attenuated vaccines and inactivated vaccines. However, they are deficient in evoking long-lasting immune response unless when administered with adjuvants [11]. Immunization 
with protein antigens alone failed to elicit $\mathrm{T}$ cell-dependent immune response unless these antigens are administered with adjuvants to completely activate $\mathrm{T}$ cells, which depend on the recognition of co-stimulators on antigen presenting cells (APCs) in addition to antigen. Adjuvants can stimulate APCs to produce co-stimulators such as CD80 and CD86 for full T-cell activation as second signals [12].

Outer membrane vesicles (OMVs) are naturally occurring bi-layered, nanospherical structures that are constitutively released by all species of Gram-negative bacteria during their normal growth both in vivo and in vitro [13-15]. As OMVs bud from the cell envelope of bacteria, they are composed of proteins, lipids, and glycans derived from the outer membrane and periplasm [13]. Except for that, vesicles produced by some bacteria harbor DNA fragments derived from the chromosome and plasmids as well as RNA [16, 17]. Due to being packaged into OMVs, these cargo molecules evade dilution and degradation and can be delivered in long distance, which may be one of the fundamentals of the interaction between bacteria and host $[16,18]$. In particular, toxins and extracellular enzymes packaged into OMVs can be delivered to host cells due to the ability of OMVs to be taken up by mammalian cells. Also, OMVs have been reported to participate in gene transfer, including the example of the transfer of antibiotic resistance by DNA in OMVs [19]. It suggests that OMVs play an important role in the evolution of bacteria in a mixed microbial environment [20]. In addition to the above functions, OMVs are also an overlooked component of the matrices of biofilms. For example, the presence and frequency of OMVs in Pseudomonas aeruginosa biofilms generated in diverse model systems and growth conditions has been demonstrated [21], and this is consistent with the findings in other bacteria. Thus, OMVs may contribute to bacterial resistance to antibiotics and environmental stress by participating in biofilm formation.

OMVs present a range of surface antigens derived from parental bacteria and have natural properties like immunogenicity, self-adjuvation and uptake by antigen presenting cells [22], in addition, owing to the presence of LPS and other pathogen-associated molecular patterns (PAMPs), OMVs are significant activators of host innate and acquired immune response pathways [23], so they can evoke humoral and cell-mediated immune responses in the host, indicating the possibility of using OMVs as vaccines against diverse pathogenic agents. Indeed, an OMV-based vaccine against serogroup B meningococcal disease has been licensed and successfully used for epidemic control, particularly in the Netherlands and Norway [24, 25]. Proven safety and efficacy records of the OMV-based vaccine demonstrate that $\mathrm{OMVs}$ can be used as vaccines to provide protective immune response and present the possibility of employing OMVs for the delivery of foreign antigens. Since the export of ClyA, a proteinaceous hemolysin expressed by E. coli, was found to be associated with OMV formation [26], increasingly more studies have shown that OMVs could be employed as a platform to deliver heterologous protein antigens of interest to confer dramatically protective efficacy utilizing ClyA as a fusion partner [27, 28].

GalT was identified as a preferentially expressed antigen during APP infection using in vivo-induced antigen technology. It has been demonstrated that GalT possesses potent immunogenicity and immune-protective efficacy, which can be considered as a potential vaccine candidate [29-31]. However, the subunit vaccine based on GalT needs protein purification and has to be administrated with adjuvants, which are time-consuming and costly. Therefore, in this study, we employed the well-characterized OMV delivery system to carry the GalT protein, with the aim of developing a convenient and efficient vaccine candidate.

\section{Materials and Methods}

\section{Animals and Ethics Statement}

Six-to-eight-week female BALB/c SPF mice (18-22 g) were purchased from Chengdu Dossy Experimental Animal Co., Ltd. The animal experiments were conducted in strict accordance with the recommendations in the China Regulations for the Administration of Affairs Concerning Experimental Animals (1988) and had been approved by the Institutional Animal Care and Use Committee of Sichuan Agricultural University (Approval Number BK2014-047), Sichuan, China.

\section{Bacterial Strains, Medium, and Culture Conditions}

E. coli $\mathrm{DH} 5 \alpha$ (Biomed, China) was used for cloning of recombinant plasmids, and E. coli JC8031 (K12 $\Delta$ tolRA) strains were used to produce the recombinant OMVs displaying GalT protein encoded by the expression vectors pBAD18-Cm. E. coli BL21 producing recombinant GalT protein was constructed in our previous study [31]. APP L20 (serovar 5b reference strain) and E. coli K-88ac31 were purchased from the China Institute of Veterinary Drug Control (China). All E. coli strains were cultured in Luria-Bertani (LB) medium. APP L20 strains were cultured in Trypticase Soy Broth (TSB) (DIFCO Laboratories, USA) with $10 \%(\mathrm{v} / \mathrm{v})$ fetal calf serum (FBS; SIJIQING, China) and nicotinamide adenine dinucleotide (NAD; $15 \mu \mathrm{g} / \mathrm{ml}$ ) at $37^{\circ} \mathrm{C}$. 
Table 1. Primers used to amplify and clone E. coli ClyA sequences and APP galT gene sequences.

\begin{tabular}{cl}
\hline Gene & \multicolumn{1}{c}{ Primer sequences $\left(5^{\prime}-3^{\prime}\right)$} \\
\hline$c l y A-\mathrm{F}$ & GCGATGGAGCTCTTAAAGAGGAGAAAGGTCATGACTGAAATCGTTGCA \\
clyA-R & CTAGTCTAGAGACTTCAGGTACCTC \\
galT $-\mathrm{F}$ & CTAGTCTAGAATGAGCCAACAATTT \\
galT $-\mathrm{R}$ & GCGATGAAGCTTCTAATGGTGATGGTGATGATGTTGATTTTTATAG \\
\hline
\end{tabular}

The underlined portions of the primer sequences were the restriction enzyme cutting sites (ClyA-F (forward): SacI; ClyA-R (reverse): XbaI; galT-F: XbaI; galT-R: HindIII).

\section{Plasmid Construction for OMVs Displaying GalT}

The recombinant plasmid was constructed as described previously [32]. To express ClyA::GalT fusion protein, a lowcopy-number integrative vector, $\mathrm{pBAD} 18-\mathrm{CM}$, was engineered to express the fusion proteins. Initially, the $c l y A$ was amplified from E. coli K-88ac31DNA. The PCR product was then digested by SacI and $\mathrm{XbaI}$ enzymes, and then cloned into a $\mathrm{PBAD} 18-\mathrm{CM}$ vector to make pBAD-ClyA. Primers targeting the galT were used on APP L20 DNA, and this product was then digested by XbaI and SalI and ligated into the $\mathrm{pBAD}-\mathrm{ClyA}$ construct to make pBAD-ClyAGalT. Primers used for amplifying the clyA/galT genes were shown in Table 1.

pBAD-GalT was electroporated into JC8031, and the transformants were selected on LB chloramphenicol medium.

\section{Preparation of OMVs}

OMVs were prepared as previously described with minor modifications [11, 28]. Briefly, E. coli JC8031 colonies were picked up and grown over in an LB medium supplemented with chloramphenicol $(25 \mu \mathrm{g} / \mathrm{ml})$. The overnight cultures were diluted 1:50 in LB medium contacting chloramphenicol and grown up to an optical density (OD600) of around 0.5 before the addition of Larabinose $(0.2 \% \mathrm{w} / \mathrm{v})$ for inducing protein expression. The culture supernatant was harvested by centrifugation after $20 \mathrm{~h}$ of induction. The culture was centrifuged at 5,000 $\times g$ for $15 \mathrm{~min}$, and then the supernatant was filtered through a $0.22-\mu \mathrm{m}$ membrane twice and concentrated by ultrafiltration using a column with a $100,000 \mathrm{KD}$ nominal molecular weight cutoff $(100,000 \mathrm{NMWC}$, Millipore). The concentrate was ultra-centrifuged at 120,000 $\times g$ for three $\mathrm{h}$ at $4^{\circ} \mathrm{C}$ (Beckman Coulter, SW32Ti Rotor). The pellet containing OMVs was resuspended in phosphate-buffered saline (PBS; $0.02 \mathrm{~mol} / 1$ phosphate buffer with $0.15 \mathrm{~mol} / 1 \mathrm{NaCl}$ at $\mathrm{pH} 7.4$ ) and then filtered through a $0.22-\mu \mathrm{m}$ membrane again, vesicles were checked for live-cell contamination by plating $20 \mu \mathrm{l}$ of preparation onto LB agar plates, then stored at $-80^{\circ} \mathrm{C}$. The concentration of total protein was determined by BCA Protein Assay Kit (Beyotime, China) according to the manufacturer's instructions.

The GalT-OMVs were visualized using electron microscopy as described [28]. Briefly vesicles were negatively stained with $2 \%$ phosphotungstic acid on 400-mesh, Formvar carbon-coated copper grids and viewed in a FEI Tecnai G2 F20 transmission electron microscope.

\section{Identification and Characterization of Engineered OMVs Displaying GalT}

Expression of the ClyA-GalT fusion protein and its content in OMVs were analyzed by SDS-PAGE and detected by western blotting. Briefly, samples were separated by SDS-PAGE and then transferred to a nitrocellulose membrane. The anti-His tag monoclonal antibody was used as a primary antibody at a 1:5000 dilution (Abmart, China), and horseradish peroxidase (HRP)coupled goat anti-mouse IgG (Bioss, China) was used as a secondary antibody at a 1:5000 dilution. The blots were developed with an ECL substrate (Bio-Rad, USA). Optical densities of the stained protein bands in SDS-PAGE were scanned to analyze the relative concentration of ClyA-GalT in recombinant OMVs using Image Lab software (Bio-Rad). The overexpressed protein band in SDS-PAGE was dug out for tandem mass spectrometry MALDITOF-TOF analysis to identify the correct expression of ClyA-GalT fusion protein and the OMVs were observed and imaged using a transmission electron microscope (Hitachi) to analyze their morphology.

\section{Immunization and Challenge}

For active immunization, six-to-eight-week mice (8 per group) were divided randomly into five groups and one group was vaccinated by subcutaneous injection with $50 \mu \mathrm{g}$ of GalT-OMVs. The control mice received empty OMVs (without target gene), purified GalT protein alone, purified GalT protein emulsified in Freund's adjuvant as a positive control and $100 \mu \mathrm{L}$ PBS buffer as a negative control, on the same program, respectively. The primary and booster immunizations were performed on day 0 and 14 . On day 28 , all mice were challenged intraperitoneally with a lethal dose of APP L20 $\left(2.5 \times 10^{7} \mathrm{CFU}\right)$. Blood samples were collected before the challenge.

For passive immunization, another five groups of eight-weekold SPF mice (8 per group) were administrated intraperitoneally with $100 \mu \mathrm{l}$ of pooled serum collected from the immunized mice $2 \mathrm{~h}$ before the challenge with APP L20. The animals were intensively monitored daily after the challenge for the presence and severity of respiratory symptoms and general illness or mortality. All the challenged mice were euthanized seven days post challenge.

\section{Humoral Immune Response}

Polystyrene microtiter 96-well plates (Costar; USA) were coated 
with purified recombinant GalT $(2.5 \mu \mathrm{g} / \mathrm{ml}$ in $0.02 \mathrm{M}$ carbonatebicarbonate buffer, $\mathrm{pH}$ 9.6), and each well was coated with $100 \mu \mathrm{l}$ of diluted protein and incubated overnight at $4^{\circ} \mathrm{C}$. The plates were blocked with $3 \%$ skim milk at $37^{\circ} \mathrm{C}$ for $1.5 \mathrm{~h}$ then washed three times using PBST buffer $(250 \mu \mathrm{l} /$ well; Solarbio, China). Serum samples were serially diluted 2-fold in blocking buffer in a range of $10^{-2}-10^{-5}$, and added to the wells (100 ul) and incubated $1 \mathrm{~h}$. The plates were then washed three times. Horseradish peroxidase (HRP)-labeled goat anti-mouse IgG was diluted (1:5000) with PBST buffer and added $(100 \mu \mathrm{l})$ onto plates for incubation at $37^{\circ} \mathrm{C}$ for $30 \mathrm{~min}$. After washing five times, soluble tetramethylbenzidine (TMB) substrate solution (Tiangen, China) was added $(100 \mu \mathrm{l})$, and the plates were incubated in the dark at room temperature for $30 \mathrm{~min}$. The reaction was terminated with $2 \mathrm{M} \mathrm{H}_{2} \mathrm{SO}_{4}$ and absorbance of each well was read at a wavelength of $450 \mathrm{~nm}$ in a microplate absorbance reader (Bio-Rad, USA).

\section{Lymphocyte Proliferation Assay and Detection of Cytokines}

Lymphocyte proliferation assays and cytokine detections were performed as previously described with minor modifications [33, 34]. Two weeks after booster immunizations, spleen lymphocytes from mice in each group were separated and cultured. Briefly, spleens were isolated aseptically and processed by gentle disruption using sterile stainless-steel screens and the rubber seal of a disposable syringe. Splenocytes were suspended in Hank's balanced salt solution (HBSS; Hyclone, USA). Red Blood Cell Lysis Buffer (Solarbio, China) was added to remove erythrocytes, and cell suspensions were centrifuged for $5 \mathrm{~min}$ at $300 \times g$. The erythrocyte-free cells were washed three times with HBSS and then resuspended in complete RPMI medium (Gibco, USA). Cell counting was performed, and $100 \mu \mathrm{l}$ of cells were added $(1 \times$ $10^{5}$ cells) into 96-well culture plates (Costar, USA). The cells were stimulated with the recombinant GalT proteins $(5 \mu \mathrm{g} /$ well) or concanavalin A (ConA; Solarbio, China) and incubated for $72 \mathrm{~h}$ at $37^{\circ} \mathrm{C}$ in a $5 \% \mathrm{CO}_{2}$ incubator. Lymphocyte proliferation assays were performed using the MTS reagent with a cell proliferation kit (Promega, USA), as per the manufacturer's instructions. The lymphocytes were incubated in 96-well culture plates with MTS reagent for $4 \mathrm{~h}$, and the absorbance was measured at $490 \mathrm{~nm}$ using an ELISA reader. To determine the expression of cytokines, the supernatants from cell cultures were harvested after $72 \mathrm{~h}$ and stored at $-80^{\circ} \mathrm{C}$. The levels of IL-2, IL- 4 , and IFN- $\gamma$ were determined in culture supernatants using an ELISA kit (Neobioscience, China), as per the manufacturer's instructions.

\section{Histopathologic and Immunohistochemical Analyses}

Lung tissues of animals from different groups were harvested and fixed with $10 \%$ neutral formalin for histopathology and immunohistochemical (IHC) analysis. Fixed tissues were sectioned at $5 \mu \mathrm{m}$ thickness and embedded in paraffin wax, then HE-stained for evaluation of histopathology using an Olympus DP71 microscope (Olympus, Japan). In IHC analysis, the sections were processed as previously described [35]. Rabbit anti-mouse myeloperoxidase (MPO) monoclonal antibody was used for IHC staining to analyze neutrophils infiltration in lung tissues from different groups. The stained IHC sections were photographed in $200 \times$ magnification and analyzed by Image-Pro Plus 6.0 software, while the integrated optical density (IOD) of each photo was determined as a positive index.

\section{Statistical Analysis}

All experimental data were expressed as mean \pm SD and analyzed by GraphPad Prism version 5.0 (GraphPad Software, $\mathrm{CA}$ ). The differences between the two groups were analyzed using Student's $t$-test, and the differences among the three groups or above were analyzed using the two-way ANOVA. P-values of $<0.05$ were considered as statistically different and were represented with an asterisk. P-values of $<0.001$ were represented by two asterisks.

\section{Results}

\section{Recombinant E. coli Strain JC8031 Produced OMVs Delivering GalT Protein}

The whole cell sample of recombinant E. coli JC8031 showed an obvious overexpressed band of approximately $75 \mathrm{KD}$ after induction with L-arabinose in comparison with a pre-induction sample (Fig. 1A). Western blotting showed that the whole cell sample after induction had a specific band, which was absent in the sample of pre-induction (Fig. 1B). Furthermore, tandem mass spectrometry confirmed the overexpressed band as ClyA-GalT fusion protein (data not shown). An electron microscopy image showed the spherical bilayer structure of OMVs, and the results showed that the outer membrane vesicles were successfully isolated with a diameter of about $20-150 \mathrm{~nm}$, which is

A

B

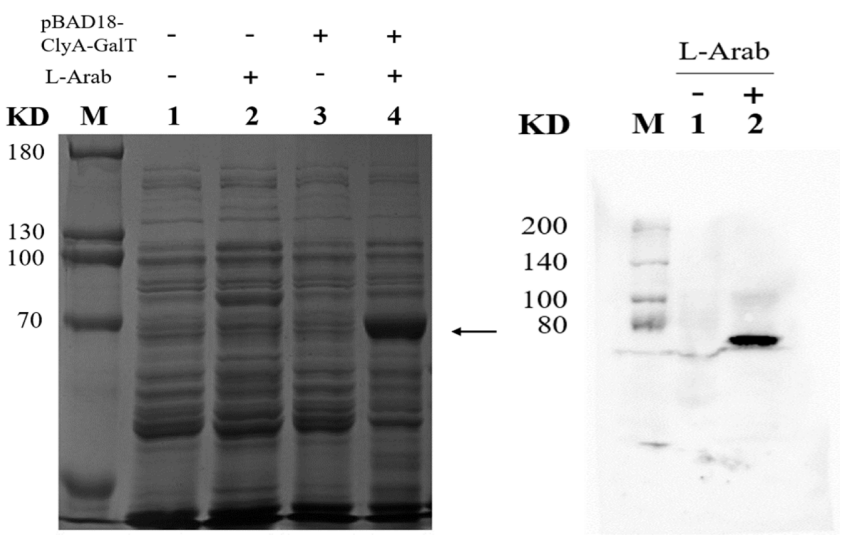

Fig. 1. SDS-PAGE (A) and western-blotting analysis (B) of JC8031 recombinant strain expressing ClyA-GalT fusion protein. 
A

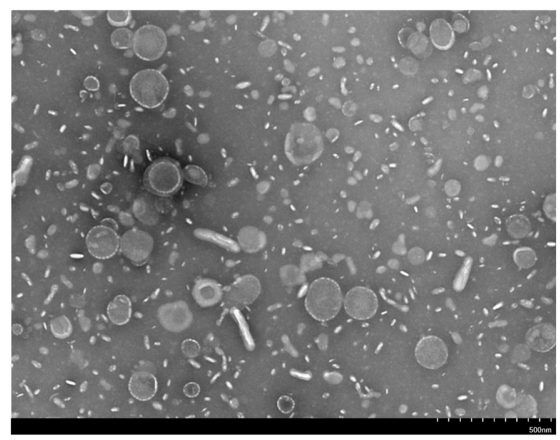

B

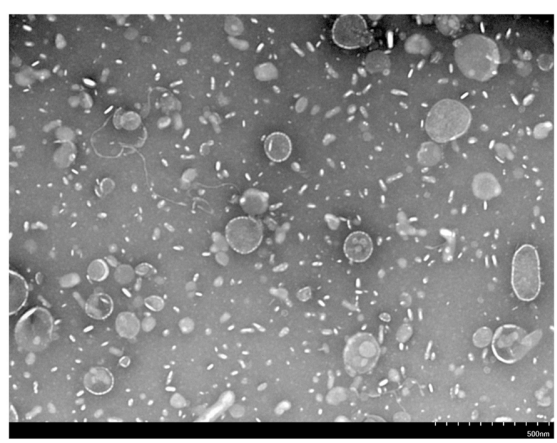

Fig. 2. Transmission electron microscope image of recombinant GalT-OMVs (A) and empty OMVs (B).

consistent with the literature reports, indicating that the introduction of the heterologous protein did not cause the outer membrane changes in vesicle morphology (Fig. 2). Also, compared with empty OMVs, recombinant GalTOMVs had a specific band, demonstrating that the GalT protein was successfully introduced into OMVs (Fig. 3).

\section{GalT-OMVs Elicited a Humoral Immune Response}

Two weeks after the booster immunization, blood samples were collected from each mouse to detect the specific antibody titers (Fig. 4). Results showed that immunization with $50 \mu \mathrm{g}$ of recombinant GalT-OMVs elicited a specific antibody titer up to $1 \times 10^{5}$, which is comparable to the antibody titer elicited by immunization with $50 \mu \mathrm{g}$ of purified GalT protein mixed with adjuvants (up to $2 \times 10^{5}$ ). Meanwhile, immunization twice with $50 \mu \mathrm{g}$ of purified GalT protein alone elicited an antibody titer of only $3 \times 10^{3}$.

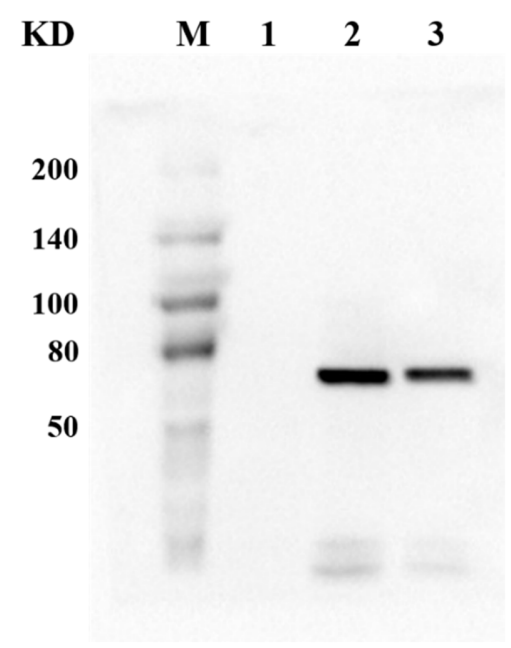

Fig. 3. Western-blotting analysis of recombinant OMVs delivering GalT protein.

Lane 1: Empty OMVs, Lane 2: GalT-OMVs, Lane 3: rGalT.
Additionally, groups immunized with empty OMVs and PBS control failed to elicit detectable specific antibody titers.

\section{GalT-OMVs Mediated a Cellular Immune Response}

After stimulation with purified recombinant GalT protein, both groups immunized with GalT-OMVs and GalT mixed with adjuvants exhibited a significant proliferative splenocytes response, while the GalT-only and vesicleimmunized groups showed no significant difference with PBS control. Similarly, strong lymphocyte proliferation was detected in each group when stimulated with ConA as a positive control (Fig. 5).

On the other hand, the levels of all three cytokines secreted in the GalT-OMV group were significantly higher than in the control groups, especially the IFN- $\gamma$. Meanwhile, in the

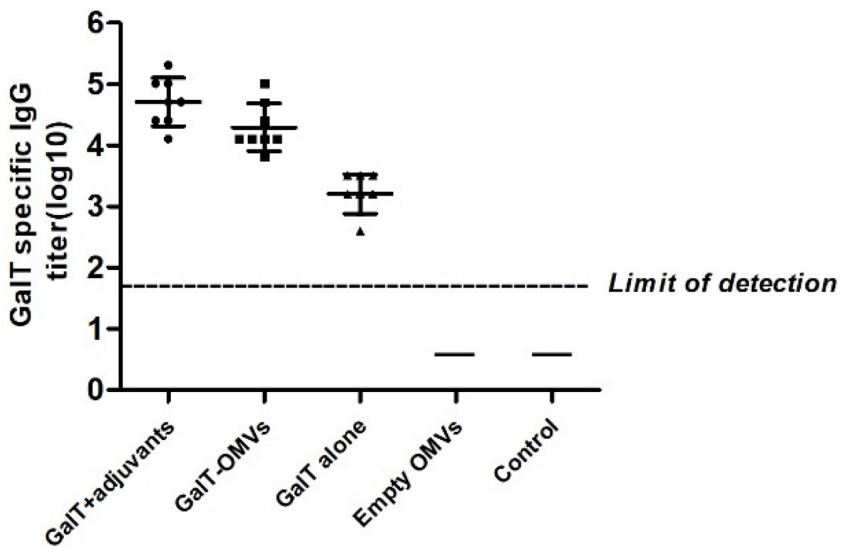

Fig. 4. Determination of specific IgG titers in serum samples collected from every mouse after booster immunization.

IgG titers were determined using indirect ELISA coated with purified recombinant GalT protein. The titer was defined as the absorbance of the highest dilution at $450 \mathrm{~nm}$ which was at least 0.1 above that of the background wells in which serum samples were replaced with PBS. 


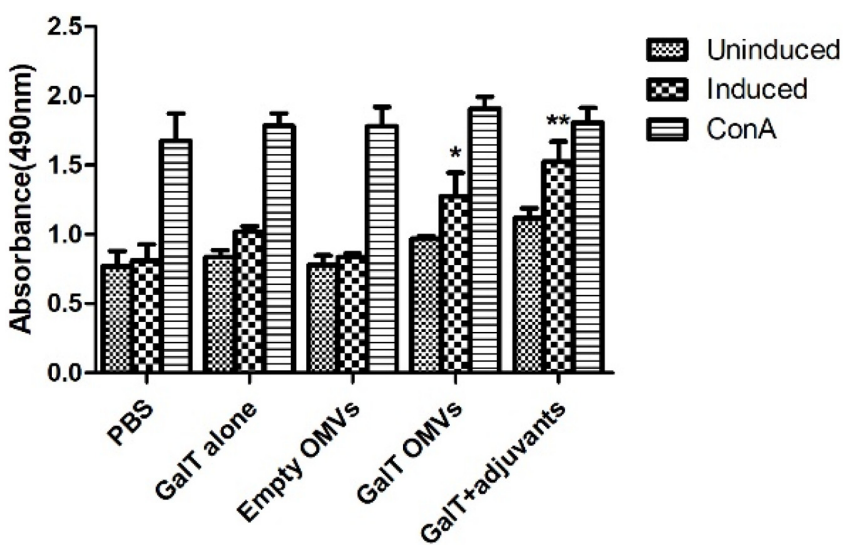

Fig. 5. Lymphocyte proliferation assay.

Splenocytes of mice two weeks after booster immunization were isolated and stimulated with recombinant GalT protein and ConA. Levels of lymphocyte proliferation in each group were detected using an MTS method and the results shown as absorbance at $490 \mathrm{~nm}$.

GalT+adjuvant group, the level of IL-4 was remarkably increased compared to the other two cytokines (Fig. 6).

\section{OMV-GalT Protein Protects Mice from APP Challenge}

As the results of vaccination and challenge experiments showed, in active immunization, susceptible/untreated mice showed severe clinical signs (labored respiration) after the APP challenge was administered. All the mice in the PBS control group died within $48 \mathrm{~h}$ after a lethal challenge with APP L20. Immunization with GalT-OMVs and GalT mixed with adjuvants provided $87.5 \%$ and $100 \%$ protection, respectively. Nevertheless, only $37.5 \%$ of mice immunized with GalT protein alone survived. As a carrier control, the survival rate of the empty OMV group was $25 \%$, indicating that OMVs derived from E. coli may provide cross-protection against APP infection to some extent (Fig. 7A). In passive immunization, all the mice receiving the administration of antisera elicited by PBS and empty OMVs died after the challenge with APP L20. 50\% and $62.5 \%$ of mice immunized passively with antisera from GalT-OMVs and GalT mixed with adjuvants were protected from the lethal APP L20 challenge, respectively (Fig. 7B).

\section{GalT-OMV-Vaccinated Animals Showed Less Severe Pathological Signs in Lung Tissues}

After the APP L20 challenge, lung tissues of mice from the PBS, GalT alone and empty OMV groups showed severe lesions. Structures of pulmonary alveoli were damaged and accompanied by the infiltration of inflammatory cells.
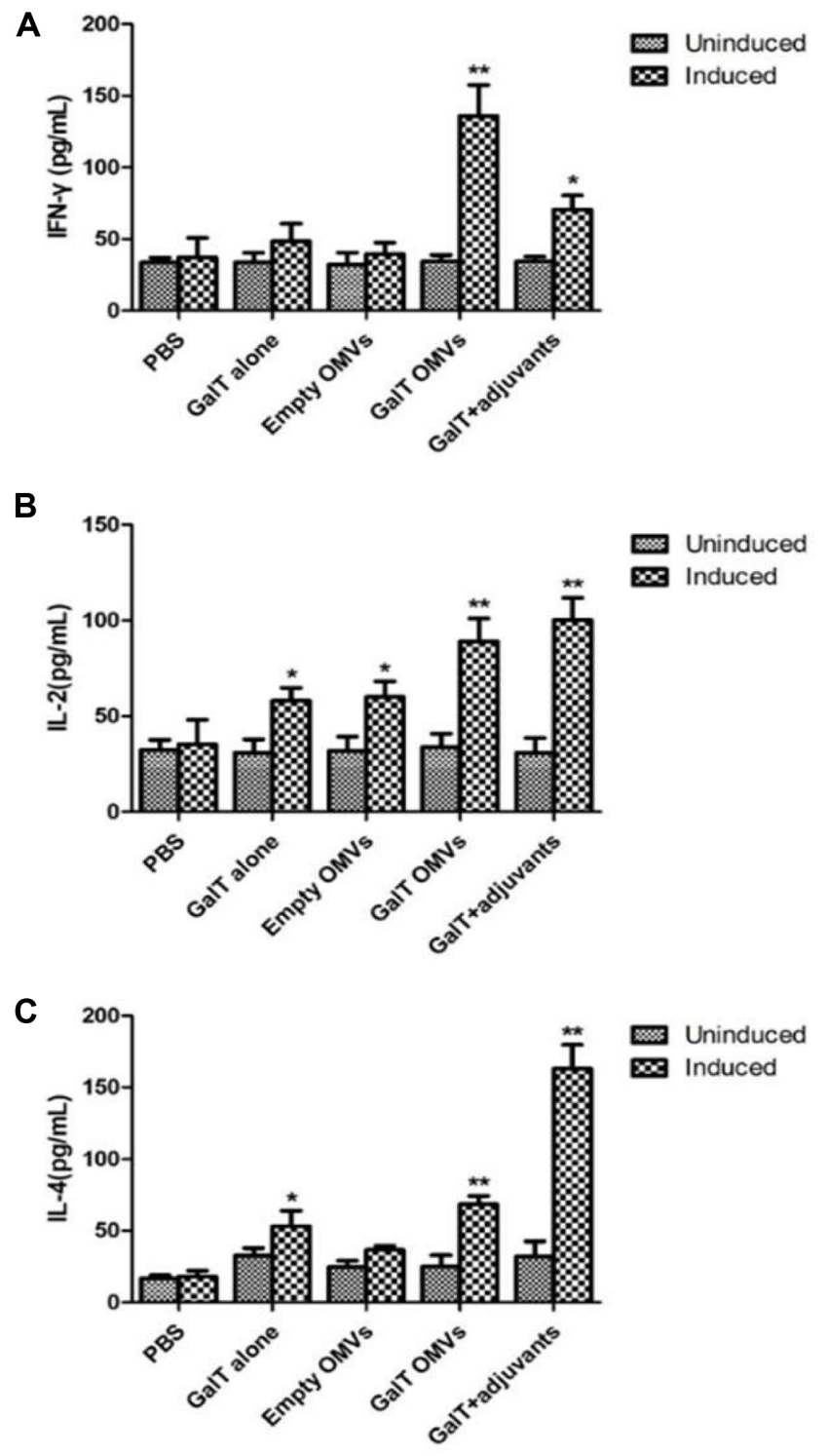

Fig. 6. The concentrations of (A) IFN- $\gamma$, (B) IL-2, (C) IL-4 in cultured spleen cells of mice.

The lung parenchyma was edematous, and expansion and congestion widely existed in the alveolar wall capillaries (Figs. 8B-8D). On the other hand, lung tissues from mice immunized with GalT-OMVs or GalT mixed with adjuvants did not appear to have significant pathological changes compared with normal control (Figs. 8E, 8F). These results indicated that immunization with recombinant GalTOMVs prevents pathological changes following challenge infection with APP and confirms that GalT-OMVs induce immune-protection against infection of APP in mouse. 
A

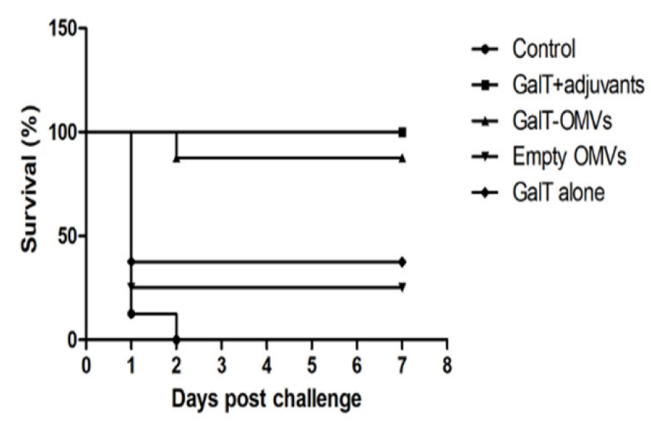

B Passive immunization

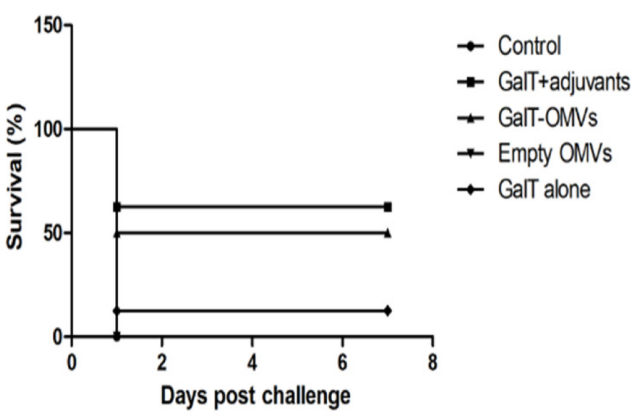

Fig. 7. The survival rate of mice challenged with a lethal dose of APP strain L20 after active immunization (A) and passive immunization (B).

\section{GalT-OMVs Prevented APP Secondary Inflammatory Response}

As the IHC analysis results of neutrophils showed, IOD of the GalT-OMV group was not significantly different compared with the normal group, as well as the GalT mixed with adjuvants group. However, the IOD of the groups immunized with PBS, GalT alone and empty OMVs was significantly higher than that for the normal group, indicating that GalT-OMVs prevented secondary inflammatory reactions and the release of toxins caused by APP colonization in the lungs after the challenge with L20 (Fig. 9).

\section{Discussion}

Vaccines play an important role in human and animal health in the fight against infectious diseases. Highefficiency vaccines that stimulate the body to produce longlasting protective immunity have the necessary conditions, such as fitting proportion, presence of pathogen-associated molecular patterns, ability antigens that stimulate the production of pathogen-specific immunity, and capacity stimulation of T-cell immune responses [36]. OMVs follow these characteristics. At present, studies have explored the

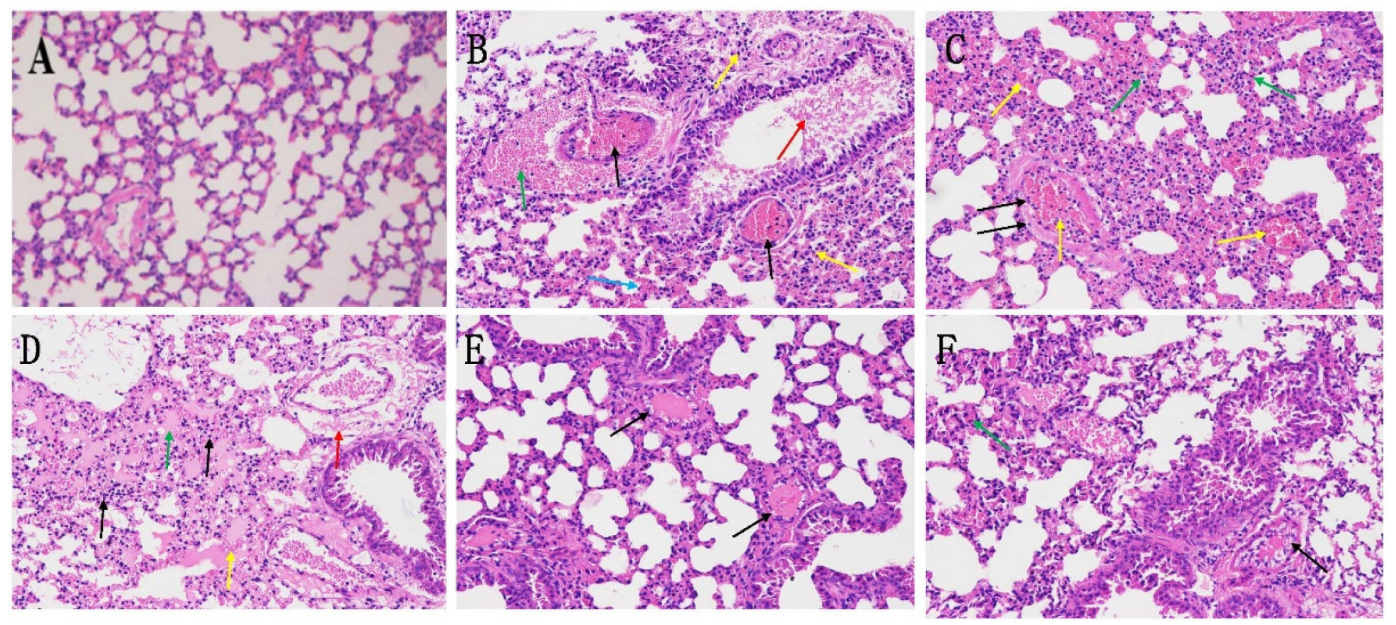

Fig. 8. Histopathologic analysis of mice lungs (200x).

Lungs of mice in each group were collected seven days after the challenge, and HE staining was used for histopathologic analysis. (A) Normal control. (B) PBS control. Lung tissue and blood vessels could be seen to swell, with congestion, loose connective tissue, and inflammatory cell infiltration. (C) GalT alone group. The vascular endothelium of the lung tissue is swollen and congested, and a small number of neutrophils are scattered in the lung tissue. (D) Empty OMV group. Local lung tissue developed edema, telangiectasia, and congestion. Simultaneously, accompanied by scattered neutrophils and short fibers. (E) GalT-OMV group. Balloon-like changes occurred in vascular endothelial cells, and no obvious lesions such as congestion, hemorrhage, and inflammation were observed. (F) Adjuvanted GalT group. Some vascular endothelial cells undergo balloon-like changes, and the alveolar wall capillaries are slightly congested. Obvious lesions have been marked with arrows. 
A
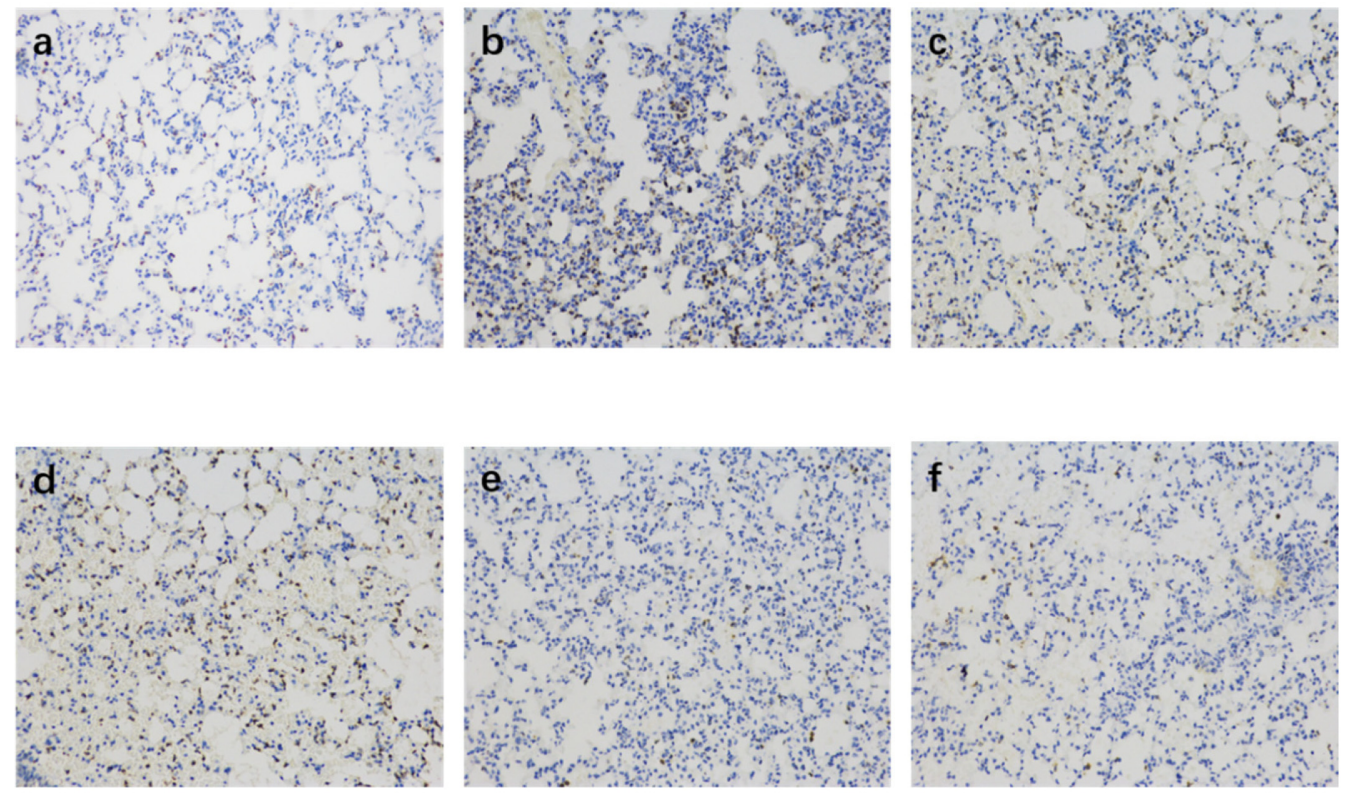

B

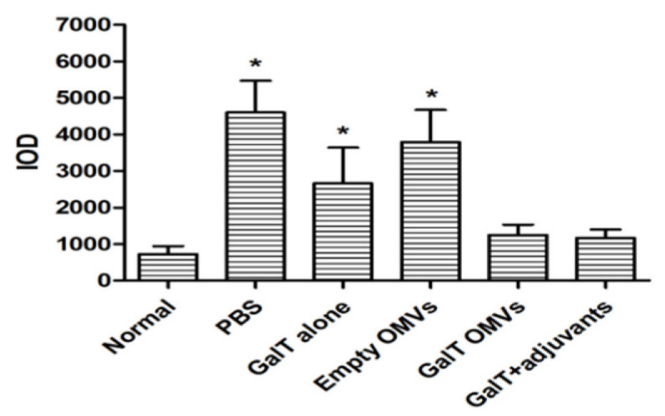

Fig. 9. Immunohistochemical analysis of neutrophils in mice lungs.

(A) Mice of each group were sacrificed post-challenge and lungs were collected for immunohistochemical analysis (200×). a. Normal lung. b. PBS control. c. GalT alone group. d. Empty OMV group. e. GalT-OMV group. f. Adjuvanted GalT group. (B) Statistical analysis of IOD of neutrophils in mice lungs.

natural OMVs secreted by Haemophilus parasuis, which belongs to the genus Pasteurella, and preliminary investigation explored its potential as a vaccine [37]. Immunization of protein as an antigen alone does not activate $\mathrm{T}$ cells, so the presence of costimulatory molecules is required. Freund's adjuvants commonly used components that stimulate antigen-presenting cells (APC) to produce costimulatory factors, such as inactivated Mycobacterium. When OMV presents GalT, it can act as an adjuvant and be swallowed by immune cells. LPS and other components on the surface can stimulate APC to produce costimulatory molecules, so GalT and costimulatory molecules are simultaneously presented to $\mathrm{T}$ cells to stimulate T-cell activation.

OMV vaccine is a popular research subject for future vaccine development because of its safety as a lifeless non- replicating vaccine and high immunogenicity with multiple antigens. Studies have shown that OMV vaccines can induce the body to produce a variety of bactericidal antibodies, and has immunoprotective capacity in animal models [38]. A variety of Gram-negative bacteria such as Bordetella pertussis [39] and Neisseria meningitidis have been used to develop new vaccines based on [40] OMVs.

In 2003, Sun [26] reported a new way of secreting protein in the intestinal tract, the OMV-mediated secretion. They found that the secretion of a pore-forming cytotoxin, ClyA, produced by intestinal bacteria does not depend on several traditional secretion systems, but accumulates in the periplasm of the bacteria, then is secreted along with OMVs sprouting from the cell membrane. Subsequently, Cornell's research team attempted to fuse green fluorescent 
protein (GFP) with ClyA to verify that ClyA could transfer GFP to OMVs[32] . Therefore, we decided to use the vesicle high-yield E.coli JC8031 as an expression bacterium, and fuse ClyA with GalT protein to induce GalT to be in the OMV.

In this study, GalT was delivered by E. coli OMVs, which proved the modification of OMVs could improve the immunogenicity of the protein. In addition, we fused the three important exotoxins ApxI, II, and III, and delivered them with E. coli OMVs, demonstrating the crossprotection ability to different serotypes of APP [41]. Furthermore, research can be extended to the use of OMVs to present antigens from different respiratory pathogens, such as the fusion of important virulence proteins of APP, H. parasuis, and P. multocida presented in the OMVs to explore whether they can be used as a broad-spectrum vaccine against multiple pathogens to generate protection.

Immune protection results have shown an interesting phenomenon. The 50 ug GalT-OMVs should contain a protein concentration of less than $50 \mathrm{ug}$ of pure GalT protein. However, the immune effect is comparable to that of the $50 \mathrm{ug}$ GalT+ adjuvant, and the empty OMV group also has a $25 \%$ protective effect. Previous studies have shown that OMVs act as bait for the immune system through multiple redirected inflammatory responses [17]. At the same time, some pathogen-associated molecular patterns (PAMPs) shared by some bacteria on the surface, such as LPS, PNAG, immunomodulatory proteins, bacterial DNA, and host pattern recognition receptor binding (PRR), may be the reason of certain cross-protection [38]. However, the use of an adjuvant as an immunostimulatory factor in combination with a vaccine stimulates the host to produce a stronger immune response against the antigen, but does not itself exhibit any antigenic properties.

In addition, the results of significantly elevated cytokine levels and previously reported OMVs produced by N. meningitidis, B. pertussis or Pseudomonas aeruginosa can stimulate epithelial TLR4 production of cytokines and activate MYD88 and NF- $\mathrm{KB}$ pathways to induce inflammatory responses, promoting immune cell maturation, and increased results of INF- $\gamma$, IL-4, IL-8 secretion [42, 43].

A major limitation in the application of OMV vaccines is the presence of lipopolysaccharide (LPS), which is known as an endotoxin. However, it is also very important for the immune-stimulatory effect of OMV and is one of the reasons for the natural adjuvant properties of OMV. The E. coli strain JC8031 used in this study to produce OMVs lacks O-antigen, which is an essential component for LPS. Thus, the adverse effect was reduced by a certain extent where no obvious lesions were found in vaccinated mice after the immunizing dose [11]. Furthermore, LPS toxicity can be reduced by further engineering the structure. It is reported that altering the acetylation structure of lipid A or knocking out the E. coli JC8031 lpxM gene both can significantly reduce LPS virulence [44, 45]. However, further work will continue to detoxify LPS as much as possible to explore the maximum safe immunized dose without decreasing the immune-protective efficacy.

In summary, our study demonstrated that OMVs could display the immune protective antigen GalT of APP and confer protection against APP virulent strain L20. Both humoral and cellular responses were elicited by recombinant GalT-OMVs, in the animal model. Furthermore, compared with purified GalT protein vaccine, recombinant GalTOMVs need only one-step purification and no supplemental adjuvants. We have demonstrated that OMVs can be used as a novel and effective platform to deliver antigens of interest for vaccines to induce immunoprotection. Further work will attempt to display fusion antigens from different porcine respiratory pathogens such as $H$. parasuis and P. multocida to develop broad-spectrum vaccines.

\section{Acknowledgments}

This research was financially supported by a grant from the Special Fund for Sichuan Application and Foundation Program (No. 2018JY0183).

\section{Conflict of Interest}

The authors have no financial conflicts of interest to declare.

\section{Reference}

1. Li Y, Cao S, Zhang L, Lau GW, Wen Y, Wu R, et al. 2016. A TolC-like protein of Actinobacillus pleuropneumoniae is involved in antibiotic resistance and biofilm formation. Front. Microbiol. 7: 1618.

2. Bosse JT, Soares-Bazzolli DM, Li Y, Wren BW, Tucker AW, Maskell DJ, et al. 2014. The generation of successive unmarked mutations and chromosomal insertion of heterologous genes in Actinobacillus pleuropneumoniae using natural transformation. PLoS One 9: e111252.

3. Cuccui J, Terra VS, Bossé JT, Naegeli A, Abouelhadid S, Li Y, et al. 2017. The N-linking glycosylation system from Actinobacillus pleuropneumoniae is required for adhesion and has potential use in glycoengineering. Open Biol. 7.

4. Zhu Z, Zhao Q, Zhao Y, Zhang F, Wen X, Huang X, et al. 
2017. Polyamine-binding protein PotD2 is required for stress tolerance and virulence in Actinobacillus pleuropneumoniae. Antonie van Leeuwenhoek 110: 1647-1657.

5. Sárközi R, Makrai L, Fodor L. 2015. Identification of a proposed new serovar of Actinobacillus pleuropneumoniae: serovar 16. Acta Vet. Hung. 63: 444-450.

6. Bossé J, Li Y, Sárközi R, Fodor L, Lacouture S, Gottschalk M, et al. 2018. Proposal of serovars 17 and 18 of Actinobacillus pleuropneumoniae based on serological and genotypic analysis. Vet. Microbiol. 217: 1-6.

7. Oldfield NJ, Donovan EA, Worrall KE, Wooldridge KG, Langford PR, Rycroft AN, et al. 2008. Identification and characterization of novel antigenic vaccine candidates of Actinobacillus pleuropneumoniae. Vaccine 26: 1942-1954.

8. Chiang CH, Huang WF, Huang LP, Lin SF, Yang WJ. 2009. Immunogenicity and protective efficacy of ApxIA and ApxIIA DNA vaccine against Actinobacillus pleuropneumoniae lethal challenge in murine model. Vaccine 27: 4565-4570.

9. Lu YC, Li MC, Chen YM, Chu CY, Lin SF, Yang WJ. 2011. DNA vaccine encoding type IV pilin of Actinobacillus pleuropneumoniae induces strong immune response but confers limited protective efficacy against serotype 2 challenge. Vaccine 29: 7740-7746.

10. Bei W, He Q, Zhou R, Yan L, Huang H, Chen H. 2007. Evaluation of immunogenicity and protective efficacy of Actinobacillus pleuropneumoniae HB04C(-) mutant lacking a drug resistance marker in the pigs. Vet. Microbiol. 125: 120-127.

11. Chen DJ, Langer R. 2010. Delivery of foreign antigens by engineered outer membrane vesicle vaccines. Proc. Natl. Acad. Sci. USA 107: 3099-3104.

12. Abbas AK, Lichtman AH, Pillai S. 2012. Basic Immunology, pp. 36-42. Ed. Saunders.

13. Schwechheimer C, Kuehn MJ. 2015. Outer-membrane vesicles from Gram-negative bacteria: biogenesis and functions. Nat. Rev. Microbiol. 13: 605-619.

14. Schertzer JW, Whiteley M. 2012. A bilayer-couple model of bacterial outer membrane vesicle biogenesis. MBio 3: 203-216.

15. Kulp A, Kuehn MJ. 2010. Biological functions and biogenesis of secreted bacterial outer membrane vesicles. Annu. Rev. Microbiol. 64: 163-184.

16. Mashburn-Warren LM, Whiteley M. 2006. Special delivery: vesicle trafficking in prokaryotes. Mol. Microbiol. 61: 839-846.

17. Kuehn MJ, Kesty NC. 2005. Bacterial outer membrane vesicles and the host-pathogen interaction. Genes Dev. 19: 2645-2655.

18. Lee EY, Choi DS, Kim KP, Yong SG. 2008. Proteomics in gram-negative bacterial outer membrane vesicles. Mass Spectrom. Rev. 27: 535-555.

19. Fulsundar S, Harms K, Flaten GE, Johnsen PJ, Chopade BA, Nielsen KM. 2014. Gene transfer potential of outer membrane vesicles of Acinetobacter baylyi and effects of stress on vesiculation. Appl. Environ. Microbiol. 80: 3469-3483.

20. Yaron S, Kolling GL, Simon L, Matthews KR. 2000. Vesicle- mediated transfer of virulence genes from Escherichia coli O157:H7 to other enteric bacteria. Appl. Environ. Microbiol. 66: 4414-4420.

21. Schooling SR, Beveridge TJ. 2006. Membrane vesicles: an overlooked component of the matrices of biofilms. J. Bacteriol. 188: 5945-5957.

22. van der Pol L, Stork M, van der Ley P. 2015. Outer membrane vesicles as platform vaccine technology. Biotechnol. J. 10: 1689-1706.

23. Ellis TN, Kuehn MJ. 2010. Virulence and immunomodulatory roles of bacterial outer membrane vesicles. Microbiol. Mol. Biol. Rev. 74: 81-94.

24. Kleijn EDD, Groot RD, Labadie J, Lafeber AB, Dobbelsteen GVD, Alphen LV, et al. 2000. Immunogenicity and safety of a hexavalent meningococcal outer-membrane-vesicle vaccine in children of 2-3 and 7-8 years of age. Vaccine 18: 1456-1466.

25. Sandbu S, Feiring B, Oster P, Helland OS, Bakke HSW, Næss LM, et al. 2007. Immunogenicity and safety of a combination of two serogroup B meningococcal outer membrane vesicle vaccines. Clin. Vaccine Immunol. 14: 10621069.

26. Wai SN, Lindmark B, Söderblom T, Takade A, Westermark M, Oscarsson J, et al. 2003. Vesicle-mediated export and assembly of pore-forming oligomers of the enterobacterial ClyA cytotoxin. Cell 115: 25-35.

27. Rappazzo CG, Watkins HC, Guarino CM, Chau A, Lopez JL, Delisa MP, et al. 2016. Recombinant M2e outer membrane vesicle vaccines protect against lethal influenza A challenge in BALB/c mice. Vaccine 34: 1252-1258.

28. Huang W, Wang S, Yao Y, Xia Y, Yang X, Li K, et al. 2016. Employing Escherichia coli-derived outer membrane vesicles as an antigen delivery platform elicits protective immunity against Acinetobacter baumannii infection. Sci. Rep. 6: 37242.

29. Zhang F, Cao S, Zhu Z, Yang Y, Wen X, Chang YF, et al. 2016. Immunoprotective efficacy of six in vivo-induced antigens against Actinobacillus pleuropneumoniae as potential vaccine candidates in murine model. Front. Microbiol. 7: 728732 .

30. Zhang F, Zhang Y, Wen X, Huang X, Wen Y, Wu R, et al. 2015. Identification of Actinobacillus pleuropneumoniae genes preferentially expressed during infection using in vivoinduced antigen technology (IVIAT). J. Microbiol. Biotechnol. 25: 1606-1613.

31. Zhang F, Zhao Q, Quan K, Zhu Z, Yang Y, Wen X, et al. 2018. Galactose-1-phosphate uridyltransferase (GalT), an in vivo-induced antigen of Actinobacillus pleuropneumoniae serovar $5 \mathrm{~b}$ strain L20, provided immunoprotection against serovar 1 strain MS71. PLoS One 13: e0198207.

32. Kim JY, Doody AM, Chen DJ, Cremona GH, Putnam D, Delisa MP. 2008. Engineered bacterial outer membrane vesicles with enhanced functionality. J. Mol. Biol. 380: 51-66.

33. Fu S, Zhang M, Xu J, Ou J, Wang Y, Liu H, et al. 2012. Immunogenicity and protective efficacy of recombinant 
Haemophilus parasuis SH0165 putative outer membrane proteins. Vaccine 31: 347-353.

34. Li M, Song S, Yang D, Li C, Li G. 2015. Identification of secreted proteins as novel antigenic vaccine candidates of Haemophilus parasuis serovar 5. Vaccine 33: 1695-1701.

35. Oh Y, Ha Y, Han K, Seo HW, Kang I, Park C, et al. 2013. Expression of leucocyte function-associated antigen-1 and intercellular adhesion molecule-1 in the lungs of pigs infected with Actinobacillus pleuropneumoniae. J. Comp. Pathol. 148: 259-265.

36. Bachmann MF, Jennings GT. 2010. Vaccine delivery: a matter of size, geometry, kinetics and molecular patterns. Nat. Rev. Immunol. 10: 787-796.

37. Mccaig WD, Loving CL, Hughes HR, Brockmeier SL. 2016. Characterization and vaccine potential of outer membrane vesicles produced by Haemophilus parasuis. PLoS One 11: e0149132.

38. Stevenson TC, Cywesbentley C, Moeller TD, Weyant KB, Putnam D, Chang YF, et al. 2018. Immunization with outer membrane vesicles displaying conserved surface polysaccharide antigen elicits broadly antimicrobial antibodies. Proc. Natl. Acad. Sci. USA 115: 201718341.

39. Asensio CJ, Gaillard ME, Moreno G, Bottero D, Zurita E, Rumbo M, et al. 2011. Outer membrane vesicles obtained from Bordetella pertussis Tohama expressing the lipid A deacylase PagL as a novel acellular vaccine candidate. Vaccine 29: 1649-1656.
40. Sierra GV, Campa HC, Varcacel NM, Garcia IL, Izquierdo PL, Sotolongo PF, et al. 1991. Vaccine against group B Neisseria meningitidis: protection trial and mass vaccination results in Cuba. NIPH Ann 14: 195-207.

41. Xu K, Zhao Q, Wen X, Wu R, Wen Y, Huang X, et al. 2018. A trivalent Apx-fusion protein delivered by E. coli outer membrane vesicles induce protection against Actinobacillus pleuropneumoniae of serotype 1 and 7 challenge in a murine model. PLoS One 13: e0191286.

42. Zhao K, Deng X, He C, Yue B, Wu M. 2013. Pseudomonas aeruginosa outer membrane vesicles modulate host immune responses by targeting the toll-like receptor 4 signaling pathway. Infect. Immun. 81: 4509-4518.

43. Fransen F, Stenger RM, Poelen MC, van Dijken HH, Kuipers B, Boog CJ, et al. 2010. Differential effect of TLR2 and TLR4 on the immune response after immunization with a vaccine against Neisseria meningitidis or Bordetella pertussis. PLoS One 5: e15692.

44. Chen L, Valentine JL, Huang CJ, Endicott CE, Moeller TD, Rasmussen JA, et al. 2016. Outer membrane vesicles displaying engineered glycotopes elicit protective antibodies. Proc. Natl. Acad. Sci. USA 113: E3609-3618.

45. Kim SH, Kim KS, Lee SR, Kim E, Kim MS, Lee EY, et al. 2009. Structural modifications of outer membrane vesicles to refine them as vaccine delivery vehicles. Biochim. Biophys. Acta 1788: 2150-2159. 